\title{
STUDIES OF HEMOLYTIC STREPTOCOCCAL INFECTION. III. THE CHARACTERISTICS OF THE HEMOLYTIC STREPTOCOCCI ISOLATED FROM PATIENTS WITH ERYSIPELAS ${ }^{1}$
}

\author{
By CHESTER S. KEEFER ANd WESLEY W. SPINK \\ (From the Thorndike Memorial Laboratory, Second and Fourth Medical Services (Harvard), \\ Boston City Hospital, and the Department of Medicine, Harvard Medical School, Boston)
}

(Received for publication September 24, 1936)

It is a matter of common experience that initial attacks or recrudescences of acute rheumatic fever frequently follow a hemolytic streptococcal infection of the throat such as acute follicular tonsillitis or scarlet fever. In the experience of Coburn and Pauli (1), recrudescences are determined by : (1) infections with a highly effective agent; (2) the disease pattern, peculiar to each rheumatic subject; (3) the intensity of the immune response of the patient as indicated by a rise in the antistreptolysin titer of the blood.

During our studies of erysipelas $(2,3)$, we did not observe a single case of rheumatic fever following the infection, and the records of 1400 cases of erysipelas that were reviewed did not reveal any cases. Other studies fail to mention erysipelas as a preceding infection in rheumatic fever, whereas it is common knowledge that hemolytic streptococcal infections of the throat frequently precede attacks. Why this should be the case is not clear; but inasmuch as Coburn and Pauli have emphasized the importance of certain characteristics of the effective organisms in the determination of recrudescences of rheumatic fever, we have studied the hemolytic streptococci from cases of erysipelas to see whether or not they differed in any respect from those isolated from rheumatic subjects.

\section{METHODS OF STUDY}

In the cases of facial erysipelas, the organisms were isolated either from the lesion or the nose. A small amount of sterile salt solution was injected into the edge of the lesion and, after waiting for several minutes, material was then aspirated and placed in blood broth. Twenty-two strains were isolated and studied, according to the following standard methods: sugar fermentation

1 This investigation was aided, in part, by a grant from the Milton Fund and Clark Bequest. with one per cent lactose, mannite, salicin, trehalose, and sorbital, final $\mathrm{pH}$ in dextrose broth, the reduction of methylene blue, the hydrolysis of sodium hippurate, hemolysis on blood agar plates (4); the type of colony, on surface plates of neopeptone agar (5); the erythrogenic toxin production, the serological classification, ${ }^{2}$ and the fibrinolysin production. Toxin-antitoxin neutralization tests were carried out when the presence of toxin was found. These results were correlated with the serological reactions that we observed in the patients.

From Table $\mathrm{I}$ it is seen that the final $\mathrm{pH}$ of all strains falls between 4.8 and 5.2. They all fermented trehalose but none fermented sorbital; none of the strains reduced methylene blue but 4 strains showed slight hydrolysis of sodium hippurate. These observations indicate that the strains were of the human type. This was confirmed by serological examinations, using the precipitin test with rabbit antiserum, as described by Lancefield (6). All of the strains fell into Group A or those of the human type.

Sugar fermentation tests with lactose, mannite, and salicin showed that, in accordance with the classification of Holman (7), there were 19 strains of Streptococcus pyogenes, 3 strains of Streptococcus infrequens and one strain of Hemolytic II in the group.

The appearance of the colonies on neopeptone blood agar plates was studied with a hand lens and with the low power of the microscope, using reflected light. Two types of colonies were observed. The one which was predominant had a smooth, shiny, convex surface; the other type of colony showed a depression in the center which.

2 We are indebted to Dr. Rebecca Lancefield of the Rockefeller Institute for Medical Research for sending us known strains of Groups $A, B$, and $C$ which we used for producing immune serum in rabbits for precipitin tests. 
TABLE I

Summary of erysipelas organisms

\begin{tabular}{|c|c|c|c|c|c|c|c|c|c|c|c|c|c|c|c|c|c|c|}
\hline \multirow{2}{*}{ 蒂 } & \multirow{2}{*}{ 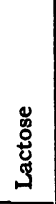 } & \multirow{2}{*}{ 苞 } & \multirow{2}{*}{ 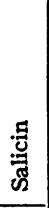 } & \multirow{2}{*}{ 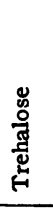 } & \multirow{2}{*}{ 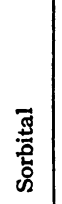 } & \multirow{2}{*}{ 空 } & \multirow{2}{*}{ 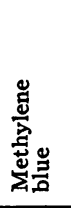 } & \multirow{2}{*}{ 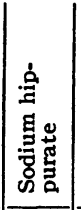 } & \multirow{2}{*}{ 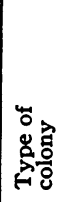 } & \multirow{2}{*}{ 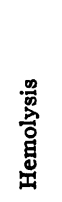 } & \multirow{2}{*}{ 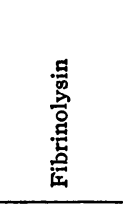 } & \multirow[b]{2}{*}{$\underset{H}{\stackrel{5}{艹}}$} & \multirow[b]{2}{*}{ 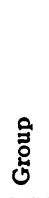 } & \multirow{2}{*}{ 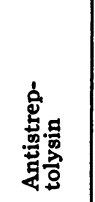 } & \multirow{2}{*}{ 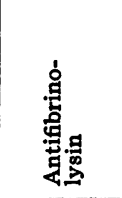 } & \multirow{2}{*}{ 焉 } & \multicolumn{2}{|c|}{$\begin{array}{l}\text { Maximum } \\
\text { number of } \\
\text { organisms } \\
\text { killed by }\end{array}$} \\
\hline & & & & & & & & & & & & & & & & & $\begin{array}{c}\mathrm{Pa}- \\
\text { tients }\end{array}$ & $\begin{array}{l}\text { Con- } \\
\text { trols }\end{array}$ \\
\hline $\begin{array}{l}1^{*} \\
2 \\
3^{*} \\
4^{*} \\
5^{*} \\
6 \\
7 \\
8^{*} \\
9 \\
10^{*} \\
11^{*} \\
12 \\
13 \\
14 \\
15^{*} \\
16 \\
17^{*} \\
18^{*} \\
19 \\
20 \\
21^{*} \\
22\end{array}$ & $\begin{array}{l}+ \\
+ \\
+ \\
+ \\
+ \\
+ \\
+ \\
+ \\
+ \\
+ \\
+ \\
+ \\
+ \\
+ \\
+ \\
+ \\
+ \\
+ \\
+ \\
+ \\
+ \\
+\end{array}$ & 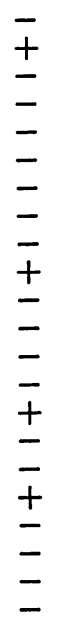 & $\begin{array}{l}+ \\
+ \\
+ \\
+ \\
+ \\
+ \\
+ \\
+ \\
+ \\
+ \\
+ \\
+ \\
+ \\
+ \\
+ \\
+ \\
+ \\
+ \\
+ \\
+ \\
+ \\
+\end{array}$ & $\begin{array}{l}+ \\
+ \\
+ \\
+ \\
+ \\
+ \\
+ \\
+ \\
+ \\
+ \\
+ \\
+ \\
+ \\
+ \\
+ \\
+ \\
+ \\
+ \\
+ \\
+ \\
+ \\
+\end{array}$ & $\begin{array}{l}z \\
z \\
z \\
z \\
z \\
z \\
= \\
= \\
= \\
= \\
= \\
= \\
= \\
= \\
= \\
=\end{array}$ & $\begin{array}{l}4.9 \\
4.8 \\
4.8 \\
5.0 \\
5.0 \\
5.2 \\
5.2 \\
5.0 \\
5.0 \\
4.8 \\
4.8 \\
4.8 \\
5.0 \\
4.8 \\
4.8 \\
5.2 \\
4.8 \\
4.8 \\
5.0 \\
4.8 \\
4.8 \\
4.8\end{array}$ & $\begin{array}{l}= \\
z \\
= \\
= \\
= \\
= \\
= \\
= \\
= \\
= \\
= \\
= \\
= \\
= \\
= \\
=\end{array}$ & $\begin{array}{l} \pm \\
\pm \\
= \\
= \\
= \\
= \\
= \\
= \\
= \\
= \\
\pm \\
= \\
\pm \\
\pm \\
\pm \\
\pm \\
\pm\end{array}$ & $\begin{array}{l}\mathbf{M} \\
\mathbf{M} \\
\mathbf{M} \\
\mathbf{M} \\
\mathbf{M} \\
\mathbf{F} \\
\mathbf{M} \\
\mathbf{M} \\
\mathbf{M} \\
\mathbf{M} \\
\mathbf{M} \\
\mathbf{M} \\
\mathbf{F} \\
\mathbf{F} \\
\mathbf{M} \\
\mathbf{F} \\
\mathbf{M} \\
\mathbf{M} \\
\mathbf{M} \\
\mathbf{M} \\
\mathbf{M} \\
\mathbf{M}\end{array}$ & $\begin{array}{l}\text { B } \\
\text { B } \\
\text { B } \\
\text { B } \\
\text { B } \\
\text { B } \\
\text { B } \\
\text { B } \\
\text { B } \\
\text { B } \\
\text { B } \\
\text { B } \\
\text { B } \\
\text { B } \\
\text { B } \\
\text { B } \\
\text { B } \\
\text { B } \\
\text { B } \\
\text { B } \\
\text { B } \\
\text { B }\end{array}$ & $\begin{array}{r}++t \\
++ \\
+++ \\
++t \\
++t \\
++t \\
++t \\
++t \\
+++ \\
+++ \\
+++ \\
++ \\
+++ \\
+++ \\
++ \\
++ \\
++ \\
+ \\
++ \\
+++\end{array}$ & $\begin{array}{r}++ \\
++ \\
++ \\
++ \\
++ \\
++ \\
++ \\
++ \\
++ \\
+ \\
+ \\
+ \\
+ \\
+ \\
+ \\
+ \\
0 \\
0 \\
0 \\
0 \\
0 \\
0\end{array}$ & $\begin{array}{l}\text { A } \\
\text { A } \\
\text { A } \\
\text { A } \\
\text { A } \\
\text { A } \\
\text { A } \\
\text { A } \\
\text { A } \\
\text { A } \\
\text { A } \\
\text { A } \\
\text { A } \\
\text { A } \\
\text { A } \\
\text { A } \\
\text { A } \\
\text { A } \\
\text { A } \\
\text { A } \\
\text { A } \\
\text { A }\end{array}$ & $\begin{array}{r}1,000 \\
3,000 \\
500 \\
500 \\
1,000 \\
500 \\
200 \\
800 \\
800 \\
800 \\
100 \\
500 \\
1,000 \\
3,000 \\
3,000 \\
500 \\
300 \\
10,000 \\
1,000 \\
500 \\
3,000 \\
1,000\end{array}$ & & $\begin{array}{l}+ \\
0 \\
0 \\
0 \\
0 \\
0 \\
0 \\
0 \\
0 \\
0 \\
0 \\
+ \\
0 \\
0 \\
0 \\
0 \\
- \\
= \\
= \\
= \\
=\end{array}$ & $\begin{array}{l}10^{-1} \\
10^{-2} \\
10^{-1} \\
10^{-1} \\
10^{-1} \\
10^{-1} \\
10^{-1} \\
10^{-1} \\
10^{-1} \\
10^{-1} \\
10^{-1} \\
10^{-2} \\
10^{-1} \\
10^{-1} \\
10^{-1} \\
10^{-4} \\
10^{-1} \\
10^{-1} \\
10^{-1} \\
10^{-1} \\
10^{-1} \\
10^{-1}\end{array}$ & $\begin{array}{l}10^{-4} \\
10^{-6} \\
10^{-7} \\
10^{-4} \\
10^{-6} \\
10^{-6} \\
10^{-6} \\
10^{-1} \\
10^{-7} \\
10^{-6} \\
10^{-6} \\
10^{-2} \\
10^{-6} \\
10^{-4} \\
10^{-4} \\
10^{-6} \\
10^{-2} \\
10^{-1} \\
10^{-1} \\
10^{-4} \\
10^{-2} \\
10^{-1}\end{array}$ \\
\hline
\end{tabular}

* Organism isolated from nose. Others isolated from the lesion.

gave it a concave appearance. The smooth shiny colonies correspond to the $\mathrm{M}$ or matt or mucoid forms, and the concave surface colonies correspond to the $F$, flocculent form of Ward and Lyons (5) or, possibly, the smooth convex form of Dawson and Olmstead (15). There were 19 strains with the $\mathrm{M}$ forms and 3 with the $\mathrm{F}$ forms. In our experience, 16 of the 19 strains grew diffusely in neopeptone broth, the other grew in a flocculent manner. Seven strains agglutinated spontaneously in plain broth, and only one strain agglutinated spontaneously in serum neopeptone.

\section{Production of skin toxin}

The toxin production of the twenty-two strains was studied by testing the capacity of each filtrate to produce an erythematous reaction in the skin of an individual who was Dick positive. The filtrate was produced by growing the organisms for 48 hours in .02 per cent dextrose-beef infusion broth and filtering it through candles. The skin of an individual who reacted positively to Dick toxin was used for testing. Dilutions of filtrate 1:100 were injected intracutaneously. Control observations were carried out with broth and heated filtrate. All readings were made at the end of 24 hours and recorded as negative, weak, or strong. The strong reactions gave an erythematous papule greater than one centimeter in diameter; a weak reaction was an erythema of one centimeter or less; a negative reaction, no reaction at all. The results are listed in Table I.

After it was determined which strains produced toxin that was powerful enough in dilutions of $1: 100$ to cause a positive skin reaction, it was determined whether these toxins could be neutralized by scarlet fever antitoxin. For these tests, the toxin was mixed with the antitoxin. From preliminary titrations with Dick toxin in a susceptible individual, it was found that $0.1 \mathrm{cc}$. of a 1:100 dilution of toxic filtrate, which we made from a scarlet fever streptococcus (NY5), was neutralized by $0.1 \mathrm{cc}$. of a diluted antiserum of a potency that contained a neutralizing capacity of 20 S.T.D. of the standard toxin. We used, therefore, the same dilution of anti-scarlet fever serum and toxic filtrates in our neutralization 
tests in studying the toxin filtrates of the erysipelas strains.

The results are presented in Table I. Nine of the strains were strong toxin producers, 7 were weak. Six strains produced no toxin. In all, then, 16 of the 22 strains, or 69 per cent, produced erythrogenic toxin, and the toxin from 14 of the 16 strains was neutralized by anti-scarlatinal antitoxin.

\section{Identification of the organisms with the Lancefield method}

Rabbits were injected with known strains of hemolytic streptococcus from Groups A, B, and $C$ so that specific antisera were obtained that would react with the group-specific (" C" substance) antigen when the precipitin test was employed. Extracts of each strain were made and tested against the antisera for precipitins, using $0.4,0.1$, and $0.025 \mathrm{cc}$. of the extract with $0.2 \mathrm{cc}$. of antiserum. The reaction was read after 15 minutes and, finally, after standing 24 hours in the ice box. From these examinations, we found that all of the organisms were members of Group A and, therefore, of the human type. The only instance in which an organism belonging to a group other than A has been isolated from a case of erysipelas is the case reported by Griffith (14) in which the organism belonged in Group C.

\section{Fibrinolysin production}

The fibrinolytic activity of the different strains was tested by the method of Tillett and Garner (8). The plasma clot from an individual who had not experienced a hemolytic streptococcal infection for two years was used, and it had been determined on many occasions that some strains of hemolytic streptococcus were capable of digesting this clot within 15 minutes.

While the strains varied in their potency when a plasma clot derived from a single source was used, this was not surprising since the patients with erysipelas showed various degrees of resistance to fibrinolysin during the course of the disease. This may be due either to the difference in individual response or to the potency of the fibrinolysin produced. The degree of resistance to fibrinolysin in the different patients is revealed in Table I. It is safe to say that most of the strains isolated from erysipelas produce fibrinolysin of high potency which is capable of exciting the production of antifibrinolytic substances in the host.

\section{Streptolysin}

We did not study the potency of the streptolysin production of the individual strains but, in view of the antistreptolysin titer of the serum that was observed in the patients infected by these strains, it is assumed that streptolysin was elaborated and there was a response on the part of the host to its production.

Ever since Fehleisen first described the streptococcus as the cause of erysipelas, there has been a lively discussion regarding the characteristics of the streptococci isolated to this particular form of infection. It has been maintained that the streptococci producing erysipelas form a special group of organisms which are distinct from those causing other forms of streptococcal infection. There is other evidence, both clinical and experimental that strains of hemolytic streptococci isolated from patients with erysipelas are made up of a number of serological types, and that they cannot be distinguished from scarlet fever strains by agglutination and absorption tests. Moreover, while it is clear that the different strains may be closely related to one another, they are also related antigenically to strains isolated from scarlet fever (9). In our experience, all strains have fallen into Group A of Lancefield's classification, although Griffith has described one strain from a case of erysipelas that belongs to Group C (Type 21, "Angel "). We have not studied the different specific types in this group of organisms.

There have been other observations which have attempted to discriminate erysipelas strains from scarlet fever and other strains of hemolytic streptococci on a basis of toxin production and toxinantitoxin neutralization tests. The results of such studies have differed somewhat in the hands of various investigators and have resulted in a divergence of opinion regarding the specificity of organisms for particular types of infection. It has been shown by Wadsworth and Coffey (10), Hooker and Follensby (11), Trask and Blake (12), and Dick and Dick (13) that there are several different toxins elaborated by toxigenic 
strains of hemolytic streptococci, and Hooker and Follensby have demonstrated two serologically distinct toxins from some single strains derived from scarlet fever. From the studies of Wadsworth and Coffey, in which it was demonstrated that toxigenic strains could be divided into several distinct groups, on a basis of toxinantitoxin neutralization tests, there was no evidence of specificity of any of the groups for a particular type of infection. That is to say, there was no evidence from this work that streptococci derived from erysipelas were specific insofar as their toxin production was concerned.

In addition to this important observation, it was shown by these same investigators that antistreptococcal sera varied markedly in valency when toxin-antitoxin neutralization tests were carried out, and this valency was dependent upon the strains used in the production of antitoxic sera. One serum had a very broad valency and neutralized 77 per cent of the toxins. This serum was made from the Dochez NY5 strain. They found two other monovalent sera which were each effective against different groups of the remaining toxins studied which represented 21 per cent of the total and, finally, there were 5 strains among 314 whose toxins were only neutralized by combining two different sera. It is important, then, in studying toxin-antitoxin neutralization tests to use monovalent sera of broad valency before concluding that the toxin production from different strains is specific for particular types of infection.

From our experience, the toxins from 14 of 16 different strains were neutralized by antistreptococcal serum ' which was made from Dochez NY5 strain. It cannot be concluded, therefore, that these tests demonstrated any specificity for these erysipelas strains insofar as toxin production was concerned.

The highest titer of the antistreptolysin in the blood of patients with erysipelas is recorded in Table I. There were wide variations but it was not uncommon to observe a vigorous response to streptolysin. The same may be said for the antifibrinolysin. Both of these tests indicate

\footnotetext{
3 This serum was kindly supplied by Dr. Elliott Robinson of the Massachusetts State Antitoxin Laboratory.
}

that patients with erysipelas respond to the various antigenic components of the hemolytic streptococcus. This is also confirmed from a study of the bactericidal tests in the patients and controls, as previously reported by us (3) and as recorded in the table.

It is perhaps worthy of comment that the blood of normal controls killed only two of the toxinproducing strains in large numbers, whereas all of the non-erythrogenic toxin-producing strains except one were killed in large numbers. This would seem to indicate that normal controls possess fewer bacteriocidins against erythrogenic toxin-producing streptococci than against the non toxin-producing strains.

\section{DISCUSSION}

On the basis of the study of the above strains of hemolytic streptococci, it seems justifiable to say that these strains are no different from those isolated from other cases of human hemolytic streptococcal infections. Moreover, we have been unable to show that these strains differ in their biological characteristics and in their grouping from the strains that are isolated from cases of tonsillitis preceding rheumatic fever. It is our belief, then, that the reason for the uncommon occurrence of rheumatic fever following erysipelas cannot be attributed to the type of infecting organism, and certainly most patients with erysipelas have a vigorous immune response as indicated by a rise in the antistreptolysin titer.

It is possible that attacks of rheumatic fever were not observed in this group of cases on account of the small number studied, or it may have been due to the fact that all of our patients were adults belonging to an age period when attacks of rheumatic fever are less common. Hodges, of the Children's Hospital in Boston, informs us that rheumatic fever was not observed following erysipelas in a group of 75 children under 12 years of age. It would be desirable to obtain more information on the incidence of rheumatic fever following erysipelas in childhood, which is the period when rheumatic fever is seen most frequently. The question of age susceptibility is a most important factor in studying rheumatic fever. Until we know more about the various 
host factors in subjects with rheumatic fever, it would seem impossible to assess the relative importance of hemolytic streptococcal infections, and the intensity of the immune response in determining the inception or recrudescences of rheumatic fever.

\section{SUMMARY AND CONCLUSIONS}

A study of 22 strains of hemolytic streptococci derived from erysipelas yielded the following results.

1. All of the strains were of human origin (Group A), as determined by the method of Lancefield.

2. Sugar fermentations, the final $\mathrm{pH}$ in dextrose broth, the reduction of methylene blue, and the hydrolysis of sodium hippurate were in accord with the findings of other workers who have studied human virulent strains.

3. All of the strains produced beta type of hemolysis on blood agar plates.

4. All of the strains but 2 produced a strong fibrinolysin.

5. Sixteen of the 22 strains produced erythrogenic toxin, and the toxin from 14 of the 16 strains was neutralized by antiscarlatinal serum that was produced from the NY5 strain of hemolytic streptococcus.

6. The blood of normal controls contained a lower bactericidal titer for the toxin-producing strains than for the non-toxin-producing strains.

7. Patients infected with these various strains developed a good immune response as indicated by the antifibrinolysin, antistreptolysin, and the bactericidal content of their blood.

8. From the available evidence, the organisms derived from erysipelas do not seem to differ from those obtained from other human infections by the hemolytic streptococcus.

We are indebted to Miss Marjorie L. Jewell and Miss Eleanor M. Fleming for technical assistance.

\section{BIBLIOGRAPHY}

1. Coburn, A. F., and Pauli, R. H., Studies on the immune response of the rheumatic subject and its relationship to activity of the rheumatic process. IV. Characteristics of strains of hemolytic streptococcus, effective and non-effective in initiating rheumatic activity. J. Clin. Invest., 1935, 14, 755.

2. Keefer, C. S., and Spink, W. W., Studies of hemolytic streptococcal infection. I. Factors influencing the outcome of erysipelas. J. Clin. Invest., 1936, $15,17$.

3. Spink, W. W., and Keefer, C. S., Studies of hemolytic streptococcal infection. II. The serological reactions of the blood during erysipelas. J. Clin. Invest., 1936, 15, 21.

4. Brown, J. H., The use of blood agar for the study of streptococci. Monograph No. 9, Rockefeller Institute for Medical Research, 1919.

5. Ward, H. K., and Lyons, C., Studies on the hemolytic streptococcus of human origin. I. Observations on the virulent, attenuated, and avirulent variants. J. Exper. Med., 1935, 61, 515.

6. Lancefield, R. C., A serological differentiation of human and other groups of hemolytic streptococci. J. Exper. Med., 1933, 57, 571.

7. Holman, W. L., The classification of streptococci. J. Med. Research, 1916, 34, 377.

8. Tillett, W. S., and Garner, R. L., The fibrinolytic activity of hemolytic streptococci. J. Exper. Med., 1933, 58, 485.

9. Stevens, F. A., and Dochez, A. R., Studies on the biology of streptococcus. VI. Biology of hemolytic streptococcus : antigenic relationships between strains of the scarlatinal and erysipelas groups. J. Exper. Med., 1926, 44, 439.

10. Wadsworth, A., and Coffey, J. M., The toxigenic properties of hemolytic streptococci from human infections. J. Immunol., 1935, 29, 505.

11. Hooker, S. B., and Follensby, E. M., Studies of scarlet fever. II. Different toxins produced by hemolytic streptococci of scarlatinal origin. J. Immunol., 1934, 27, 177.

12. Trask, J. D., and Blake, F. G., Heterologous scarlet fever. J. A. M. A., 1933, 101, 753.

13. Dick, G. F., and Dick, G. H., Specificity of soluble toxins produced by hemolytic streptococci. J. A. M. A., 1929, 93, 1784.

14. Griffith, F., Serological classification of Streptococcus pyogenes. J. Hyg., 1934, 34, 542.

15. Dawson, M. H., and Olmstead, M., Mucoid phase of Streptococcus hemolyticus. Science, 1934, 80, 296. 